\title{
Disk-assisted Spin-down of Young Radio Pulsars
}

\section{Citation}

Menou, Kristen, Rosalba Perna, and Lars Hernquist. 2001. “Disk-Assisted Spin-down of Young Radio Pulsars." The Astrophysical Journal 554 (1): L63-66. https://doi.org/10.1086/320927.

\section{Permanent link}

http://nrs.harvard.edu/urn-3:HUL.InstRepos:41381658

\section{Terms of Use}

This article was downloaded from Harvard University's DASH repository, and is made available under the terms and conditions applicable to Other Posted Material, as set forth at http:// nrs.harvard.edu/urn-3:HUL.InstRepos:dash.current.terms-of-use\#LAA

\section{Share Your Story}

The Harvard community has made this article openly available.

Please share how this access benefits you. Submit a story.

Accessibility 


\title{
Disk-Assisted Spindown of Young Radio Pulsars
}

\author{
Kristen Menou, ${ }^{1}$ \\ Princeton University, Department of Astrophysical Sciences, Princeton NJ 08544, USA, \\ kristen@astro.princeton.edu \\ Rosalba Perna ${ }^{2}$ and Lars Hernquist \\ Harvard-Smithsonian Center for Astrophysics, 60 Garden Street, Cambridge MA 02138, USA, \\ rperna@cfa.harvard.edu, lars@cfa.harvard.edu
}

\begin{abstract}
We present a model for the spindown of young radio pulsars in which the neutron star loses rotational energy not only by emitting magnetic dipole radiation but also by torquing a surrounding disk produced by supernova fallback. The braking index predicted in our model is in general less than $n=3$ (the value for pure dipole magnetic radiation), in agreement with the reported values of $n<3$ for five young radio pulsars. With an accuracy of $30 \%$ or better, our model reproduces the age, braking index and third frequency derivative of the Crab pulsar for a disk mass inflow rate in the range $3 \times 10^{16}-10^{17} \mathrm{~g} \mathrm{~s}^{-1}$.
\end{abstract}

subject headings: accretion, accretion disks - supernovae: general - pulsars: general - stars: neutron

\section{Introduction}

The magnetic dipole model for radio pulsars has proven extremely successful in explaining the observational properties of these objects (Pacini 1967; 1968; Gunn \& Ostriker 1969; Gold 1968; Goldreich \& Julian 1969; see, e.g., Shapiro \& Teukolsky [1983] for an overview; Manchester \& Taylor [1977] for a detailed review). A longstanding problem of the theory, however, has been that the predicted braking index (see $\S 2.1$ for definition) is $n=3$, while measurements of braking indices in five young radio pulsars are all less than 3 (see $\S 3$ for the most recent measured values). Various effects have been proposed to explain this discrepancy, such as magnetic axis wandering (Macy 1974), non-standard vacuum dipole theory (Melatos 1997), and non-dipolar field structure or field strength secular evolution (Blandford, Applegate \& Hernquist 1983; Blandford \& Romani 1988).

\footnotetext{
${ }^{1}$ Chandra Fellow

${ }^{2}$ Harvard Junior Fellow
} 
In this Letter, we explore the consequences of a recent proposal by Chatterjee, Hernquist \& Narayan (2000) according to which young radio pulsars could be surrounded by remnant disks produced by supernova fallback (see also Michel \& Dessler 1981, 1983; Michel 1988; Yusifov et al. 1995; Alpar 1999, 2000). The disk may indeed be able to contribute to the neutron star spindown even in the radio pulsar phase, provided that matter does not fall through the magnetosphere inside the light cylinder radius. We note that Marsden, Lingenfelter \& Rothschild (2001a,b) have recently considered some of the consequences of the presence of such a disk around a radio pulsar, with a special emphasis on explaining discrepancies between a pulsar's true age and its timing age (see also Gvaramadze [2001] for the case of an interaction with dense circumstellar material). The outline of this Letter is as follows. In $\S 2$, we derive a theory for pulsar spindown in the presence of a fallback disk and the associated propeller torques. We apply the theory to the Crab pulsar and other young radio pulsars in $\S 3$.

\section{Pulsar Spindown Model}

We certainly lack a proper understanding of the magnetospheric interaction between a radio pulsar and a surrounding disk. However, simple arguments can be used to derive a reasonable rate at which the pulsar is spun down by this interaction. Implicit to our model is the assumption that the disk can survive the high-energy environment created by the intense relativistic pulsar wind. For the pulsar mechanism to operate, we require the disk to be located beyond the light cylinder radius, at $R_{l c}=c / \Omega$ (where $\Omega$ is the neutron star angular speed and $c$ is the speed of light). On the other hand, the magnetic field lines are open beyond $R_{l c}$ and the field structure is no longer dipolar. This is the main motivation for our assumption that the disk extends down to $R_{l c}$. At $R_{l c}$, the inflowing material encounters a dipolar field structure rotating at nearly the speed of light: an efficient propeller effect (Illarionov \& Sunyaev 1975) may be expected to operate there, spinning down the neutron star and preventing mass inside the pulsar magnetosphere at the same time. $]^{3}$

Following Menou et al. (1999; see also Daumerie 1996), we adopt an efficient propeller torque

$$
\dot{J} \equiv I \dot{\Omega}=-2 \dot{M} R_{m}^{2} \Omega,
$$

where $I$ is the moment of inertia of the neutron star, a dot denotes a time derivative and $\dot{M}$ is the disk mass inflow rate at the magnetospheric radius, $R_{m}$. This expression for the torque assumes that the material flung away by the propeller effect has been accelerated to an angular speed corresponding to that of the star. In the pulsar case, we effectively take $R_{m}=R_{l c}$ and the material flung away only approaches the speed of the light. Under these simple assumptions, the

\footnotetext{
${ }^{3}$ We note that, if the disk were truncated beyond $R_{l c}$ (e.g. because of a large energy density right outside the light cylinder), the torque on the neutron star would likely be largely reduced or entirely suppressed.
} 
torque for this mildly relativistic propeller is given by

$$
\dot{J}=-2 \dot{M} c^{2} \Omega^{-1}
$$

which is essentially what one expects from dimensional arguments assuming the torque acts at $R_{l c}$. A more efficient, relativistic propeller is energetically possible; it would correspond to a reduced value of the disk mass inflow rate for a given value of the torque.

The pulsar loses rotational energy at a rate:

$$
\dot{E} \equiv I \Omega \dot{\Omega}=-\beta \Omega^{4}-\gamma
$$

where the first term on the RHS corresponds to magnetic dipole radiation losses and the second term to propeller losses. We have

$$
\gamma=2 \dot{M} c^{2} \approx 2 \times 10^{37} \operatorname{erg~s}^{-1}\left(\frac{\dot{M}}{10^{16} \mathrm{~g} \mathrm{~s}^{-1}}\right),
$$

and

$$
\beta=\frac{B_{p}^{2} \sin ^{2} \alpha R_{N S}^{6}}{6 c^{3}},
$$

where $R_{N S}$ is the neutron star radius and $B_{p}$ is the magnetic field strength at the magnetic pole, inclined at an angle $\alpha$ from the rotation axis (see, e.g., Shapiro \& Teukolsky 1983).

In what follows, we assume that Eq. (3) is valid at all times. We note that this assumption is not entirely consistent with the evolutionary scenario of Chatterjee et al. (2000), in which a neutron star decouples completely from its surrounding fallback disk when it enters the radio pulsar phase. We expect this discrepancy to be minor given that magnetic dipole radiation still dominates the losses in the present model. More importantly, a young neutron star is not initially in the radio pulsar phase in the Chatterjee et al. model, but in a "dim propeller" phase (during which the magnetospheric radius has not yet reached the light cylinder radius). This phase can last up to several hundreds of years in the Chatterjee et al. model and it is not captured by our Eq. (3). This discrepancy suggests that the results derived below for the integrated neutron star spin history should be interpreted with caution (instantaneous quantities such as the braking index are not directly affected, however).

\subsection{Steady mass inflow rate}

From Eq. (3), we find that the braking index is

$$
n \equiv \frac{\ddot{\Omega} \Omega}{\dot{\Omega}^{2}}=3-\frac{1}{\frac{1}{4}+\frac{\beta}{4 \gamma} \Omega^{4}},
$$

if $\gamma$ (i.e. the disk mass inflow rate) is constant with time. The breaking index is therefore less than 3 , the value corresponding to pure magnetic dipole radiation losses (recovered in the limit $\gamma \rightarrow 0$ ). 
Following the usual procedure, Eq. (3) can be separated and integrated to constrain the neutron star spin history. We rewrite Eq. (3) as

$$
\frac{d \Omega^{2}}{1+\frac{\beta}{\gamma} \Omega^{4}}=-\frac{2 \gamma}{I} d t
$$

which can be integrated (assuming $\gamma=$ constant) by noting that the LHS is of the form $d y /\left(1+y^{2}\right)=d(\arctan (y))$. This yields, at any time $t$ after the system's birth:

$$
t=\frac{I}{2(\gamma \beta)^{1 / 2}}\left[\arctan \left[\left(\frac{\beta}{\gamma}\right)^{1 / 2} \Omega_{i}^{2}\right]-\arctan \left[\left(\frac{\beta}{\gamma}\right)^{1 / 2} \Omega^{2}(t)\right]\right],
$$

where $\Omega(t)$ is the angular rotation speed at time $t$ and $\Omega_{i}$ is the angular rotation speed at birth. In the limit of very large $\Omega_{i}$, the left arctan is simply equal to $\pi / 2$. There is no simple relation between the pulsar timing age and true age in this solution, contrary to the case of pure magnetic dipole radiation losses (Shapiro \& Teukolsky 1983; Gunn \& Ostriker 1969).

\subsection{Time-dependent mass inflow rate}

We generalize here the above calculations to the case of a mass inflow rate decreasing with time, as expected in the fallback disk scenario (Chatterjee et al. 2000; Menou, Perna \& Hernquist 2001). Allowing the mass inflow rate to vary with time, the braking index becomes:

$$
n=3-\frac{1-\frac{1}{4} \frac{\dot{\gamma}}{\gamma} \frac{\Omega}{\dot{\Omega}}}{\frac{1}{4}+\frac{\beta}{4 \gamma} \Omega^{4}}
$$

where both $\dot{\gamma}$ and $\dot{\Omega}$ are negative (assuming a decreasing mass inflow rate). The quantity $-\Omega / \dot{\Omega}$ is twice the usual pulsar timing age $(\equiv P / 2 \dot{P})$, while the quantity $-\alpha \gamma / \dot{\gamma}$ is actually the true age of the system if, as expected, the mass inflow rate obeys a power law evolution in time of the form $\gamma(t) \propto t^{-\alpha}$.

While the braking index and the second deceleration parameter (defined in §3.1) are instantaneous quantities which can easily be computed for any power-law solution (using, if known, the values of the true age and the timing age as explained above), the spin history cannot be computed analytically for an arbitrary power law index $\alpha$, but only in some special cases.

For simplicity, here we restrict our discussion to solutions for the neutron star spin history with a time-dependent mass inflow rate satisfying $\gamma(t)=\gamma_{0}\left(t / t_{0}\right)^{-2}$. Let $w=1 / \Omega^{2} t, a=2 \beta / I$ and $b=2 \gamma_{0} t_{0}^{2} / I$, then the differential equation (3) becomes

$$
w+t \frac{d w}{d t}=a+b w^{2}
$$

\footnotetext{
${ }^{4}$ We note that this expression for the braking index can potentially yield values of $n>3$.
} 
This can be separated and integrated:

$$
\int \frac{d w}{b w^{2}-w+a}-\ln t=\text { constant }
$$

If $4 a b<1$ then the integration yields

$$
\frac{1}{c} \ln \left|\frac{2 b w-1-c}{2 b w-1+c}\right|-\ln t=\text { constant }
$$

where $c=\sqrt{1-4 a b}$. The constant is fixed by the value of the variable $w$ at some initial time $t_{i}$. The solution for $\Omega$ can then be easily derived:

$$
\Omega^{2}=\frac{2 b}{t} \frac{\left(2 b w_{i}-1+c\right)-\left(2 b w_{i}-1-c\right)\left(t / t_{i}\right)^{c}}{(1+c)\left(2 b w_{i}-1+c\right)-(1-c)\left(2 b w_{i}-1-c\right)\left(t / t_{i}\right)^{c}} .
$$

At later times, $t \gg t_{i}$, and the solution can be well approximated by

$$
\Omega^{2} \approx \frac{1+c}{2 a t} .
$$

Evaluating the above equation at the present time $t_{0}$ yields

$$
t_{0}=\frac{I}{2 \beta \Omega_{0}^{2}\left(1+\frac{\gamma_{0}}{\beta \Omega_{0}^{4}}\right)} .
$$

Note that the factor $I /\left(2 \beta \Omega_{0}^{2}\right)$ is the pulsar timing age (in the case of pure magnetic dipole radiation losses). Although this solution corresponds to rather simple evolution laws for $\Omega$ and $\gamma$, it is useful as an illustration of the effect of allowing for a time-varying mass inflow rate in the equations (as compared to the steady case considered in $\$ 2.1$ ). Given that a fallback disk should have a mass inflow rate decreasing approximately as $t^{-1.2}$ (Cannizzo et al. 1990; Chatterjee et al. 2000; Menou et al. 2001), the steady and simple time-varying cases considered here may be expected to bracket the real case $\left(\gamma \propto t^{-1.2}\right)$, for which a numerical integration is required in full generality.

\section{Applications}

\subsection{The Crab Pulsar}

For the Crab pulsar (PSR B0531+21), we adopt $I \approx 10^{45} \mathrm{~g} \mathrm{~cm}^{-2}$ and $\beta=3.85 \times 10^{29} \mathrm{erg} \mathrm{s}^{3}$. These values are derived from measurements of $\Omega, \dot{\Omega}$, the estimated value of $-\dot{E} \approx 5 \times 10^{38} \mathrm{erg} \mathrm{s}^{-1}$ and the assumption of pure magnetic dipole radiation losses (see Shapiro \& Teukolsky 1983;

Taylor, Manchester \& Lyne 1993). The propeller losses are comparatively small in the applications considered below and for the Crab pulsar:

$$
\frac{\beta \Omega^{4}}{\gamma} \approx 25\left(\frac{\dot{M}}{10^{16} \mathrm{~g} \mathrm{~s}^{-1}}\right)^{-1} .
$$


We recall here that, in 1972, the true age of the Crab pulsar was 918 yrs, while its timing age was measured to be 1243 yrs (which is half the characteristic age as defined by Shapiro \& Teukolsky 1983). The braking index of the Crab pulsar is $n=2.509$ (e.g. Lyne et al. 1988).

In the case of steady mass inflow in the fallback disk outside the light cylinder, we find using Eq. (间) that the braking index is $n=2.85,2.57$ and 1.86 for the mass inflow rates $\dot{M}=10^{16}$, $3 \times 10^{16}$ and $10^{17} \mathrm{~g} \mathrm{~s}^{-1}$, respectively. For the same mass inflow rates, Eq. (8) predicts a system age of $t_{0}=1130,1101$ and $1018 \mathrm{yrs}$, respectively (in the limit of very large initial angular speed $\Omega_{i}$ ).

In the case of a mass inflow rate decreasing with time as $t^{-1.2}$, we find using Eq. (9) that the braking index is $n=2.97,2.93$ and 2.78 for the (current) mass inflow rates $\dot{M}=10^{16}, 3 \times 10^{16}$ and $10^{17} \mathrm{~g} \mathrm{~s}^{-1}$, respectively. The age cannot be simply computed with the power law index $\alpha=1.2$. However, it will be bracketed by the values obtained for steady mass inflow above and the $\alpha=2$ case, for which Eq. (15) holds. For the same (current) mass inflow rates as above, Eq. (15) predicts a system age of $t_{0}=1099,1020$ and $817 \mathrm{yrs}$, respectively.

In each of the cases above (steady mass inflow and $\gamma \propto t^{-2}$ ), it is possible to find a mass inflow rate for which both the Crab pulsar true age and braking index are predicted to within $20 \%$ or better. Consequently, it appears that a fallback disk with a current mass inflow rate in the range $3 \times 10^{16}-10^{17} \mathrm{~g} \mathrm{~s}^{-1}$ can account for the properties of the Crab pulsar with an accuracy of $20 \%$ or so.

The model can be further tested with the third time derivative of the Crab pulsar frequency (Lyne et al. 1988; Blandford \& Romani 1988). It is useful to define a second deceleration parameter:

$$
p \equiv \frac{\dddot{\Omega} \Omega^{2}}{\dot{\Omega}^{3}}=6+\left(3-\frac{1}{\frac{1}{4}+\frac{\beta \Omega^{4}}{4 \gamma}}\right)^{2}-\frac{1+\frac{\dot{\gamma}}{4 \gamma} \frac{\Omega}{\Omega}}{\frac{1}{4}+\frac{\beta \Omega^{4}}{4 \gamma}}+\frac{\frac{\ddot{\gamma}}{\gamma} \frac{\Omega^{2}}{\dot{\Omega}^{2}}-\frac{\dot{\gamma}}{\gamma} \frac{\Omega}{\dot{\Omega}}}{1+\frac{\beta \Omega^{4}}{\gamma}},
$$

which has the correct limit $p=15$ for $\gamma \rightarrow 0$ (pure magnetic dipole radiation). The expression for steady mass inflow is found by forcing $\ddot{\gamma}=\dot{\gamma}=0$ in the above equation. Note again that $\alpha(\alpha+1) \gamma / \ddot{\gamma}$ is the square of the pulsar true age for a power law evolution of the disk mass inflow rate $\left(\gamma(t) \propto t^{-\alpha}\right)$, while $-\Omega / \dot{\Omega}$ is twice the pulsar timing age. The measured value of $p$ for the Crab is $\approx 10.2$ (Lyne et al. 1988; Blandford \& Romani 1988; Melatos 1997). We find, for the case of steady mass inflow, that the equation above predicts $p=12.6$ for $\dot{M}=3 \times 10^{16} \mathrm{~g} \mathrm{~s}^{-1}$ and $p=9.46$ for $\dot{M}=10^{17} \mathrm{~g} \mathrm{~s}^{-1}$. For a time dependent evolution with $\alpha=1.2$, we find $p=13.6$ and $p=11.96$ for the same mass inflow rates, respectively. The second deceleration parameter is therefore correctly predicted by the model (with an accuracy of $30 \%$ or so) for fallback disk mass inflow rates in the range $3 \times 10^{16}-10^{17} \mathrm{~g} \mathrm{~s}^{-1}$. 


\subsection{Other Young Radio Pulsars}

Braking indices have been measured for four other young radio pulsars. The measured values are $n=2.2 \pm 0.1,1.4 \pm 0.2,2.91 \pm 0.05$ and 2.837 \pm 0.001 for PSR B0540-69, PSR B0833-45 (Vela), PSR J1119-6127 and PSR B1509-58, respectively (Lyne, Pritchard \& Graham-Smith 1993; Deeter, Nagase \& Boynton 1999; Lyne et al. 1996; Kaspi et al. 1994; Camilo et al. 2001).

It is possible to calculate, within the framework of our pulsar spindown model, the disk mass inflow rates required in each system to account for the measured breaking indices. In the absence of known true ages for these four pulsars, we calculate theoretical braking indices in two limits: (1) the steady mass inflow case (Eq. 6) and (2) the time-dependent case (Eq. 9) with the additional simplifying assumption that the pulsar timing age is a correct measure of the pulsar true age (the validity of this assumption, appropriate for the magnetic dipole model, was recently challenged by Gaensler \& Frail 2000). The pulsar properties are taken from Taylor et al. (1993) and Camilo et al. (2001).

We find that the pulsar spindown model can account for the braking indices of PSR B0540-69, PSR J1119-6127 and PSR B1509-58 for disk mass inflow rates $\sim 3 \times 10^{16}$, $10^{14}$ and $\lesssim 10^{15} \mathrm{~g} \mathrm{~s}^{-1}$, respectively. In each case, the propeller losses represent a reasonably small fraction of the total pulsar rotational losses. The very small value of $n \approx 1.4$ for the Vela pulsar cannot be accounted for by a model in which propeller losses are small. We note that the sub-Eddington mass inflow rates required for PSR B0540-69, PSR J1119-6127 and PSR B1509-58 (as well as the Crab) appear consistent with the values expected at late stages (before the fallback disk becomes neutral) in the evolutionary scenario described by Menou et al. (2001). One expects the magnetospheric interaction to be largely reduced or even suppressed when the gaseous disk becomes neutral (independently of the passive-disk outcome advocated in Menou et al. 2001).

\subsection{Observational Constraints on Disk Sizes}

The presence of disks around pulsars cannot be hidden: even a relatively compact disk will produce some optical emission, as radiation at these wavelengths is produced at relatively small radii.

Optical emission is a common feature of pulsars: since the first detection of the Crab in 1969, several more pulsars have been observed in the optical bandpass, and some of them have been seen to pulsate. In the cases where no pulsations are seen, the optical radiation is believed to be mainly of thermal origin, while pulsed optical emission is generally considered to be either a mixture of thermal and non-thermal (magnetospheric) radiation, or purely magnetospheric. Pacini (1971) and Pacini \& Salvati (1987) proposed that the high energy emission comes from relativistic electrons radiating via synchrotron processes in the outer regions of the magnetosphere.

The Crab, Vela, and PSR B0540-69 pulsars belong to the above class, all possessing a 
pulsating optical counterpart. On the other hand, no pulsations have been detected for the candidate optical counterpart of the source PSR B1509-58 proposed by Caraveo et al. (1994).

In the following, we estimate, for each of the sources that we consider (except for PSR J1119-6127, for which no optical limits are available so far), the maximum size allowed for a disk to be compatible with the observed optical limits. For the three sources for which the optical emission is pulsed, we consider as an upper limit the lowest value of the emission (i.e. the minimum of the light curve), while for PSR B1509-58 we consider the detected value, assuming that it is indeed associated with the pulsar.

The inner radius of the disk is assumed to coincide with the light cylinder radius, $R_{l c}$. The disk model that we adopt is similar to that described by Perna, Hernquist \& Narayan (2000). We include both the flux due to viscous dissipation and that due to reradiation. The irradiating X-ray luminosity is assumed to be isotropic, because, due to light bending in the vicinity of the star, beaming effects are smoothed out (Perna \& Hernquist 2000).

Using the X-ray luminosities and periods from observations (see Becker \& Trumper 1997 for a review), we find (assuming a disk inclination angle of $60^{\circ}$ ) that, for a mass inflow rate $\sim 10^{17} \mathrm{~g}$ $\mathrm{s}^{-1}$ a disk of radial extent $\lesssim 6 \times 10^{9} \mathrm{~cm}$ is compatible with the optical limits for the Crab pulsar. A large disk $\left(Z 10^{13} \mathrm{~cm}\right)$ is allowed for both PSR B0540-69 and PSR B1509-58 at the mass inflow rate of $\sim 3 \times 10^{16}$ and $\sim 10^{15} \mathrm{~g} \mathrm{~s}^{-1}$ respectively, while for Vela only a very small disk $\left(\lesssim 10^{9} \mathrm{~cm}\right)$ at an high inclination $\left(z 89^{\circ}\right.$ ) would be compatible with the observational constraints ${ }^{6}$. The allowed size becomes larger as $\dot{M}$ decreases. Again, we note that, with the exception of the Vela pulsar, these disk sizes appear consistent with the values expected at late stages (before the fallback disk becomes neutral) in the evolutionary scenario of Menou et al. (2001).

Support for this work was provided by NASA through Chandra Fellowship grant PF9-10006 awarded by the Smithsonian Astrophysical Observatory for NASA under contract NAS8-39073.

\section{REFERENCES}

Alpar, M.A. 1999, preprint, astro-ph/9912228

Alpar, M.A. 2000, ApJ, submitted, astro-ph/0005211

Becker, W. \& Trumper 1997, A\&A, 326, 682

Blandford, R.D., Applegate, J.A. \& Hernquist, L. 1983, MNRAS, 204, 1025

\footnotetext{
${ }^{5}$ The interpretation of this optical counterpart is however a bit controversial (Mignani et al. 1998; Chakrabarty \& Kaspi 1998; Gvaramadze 2001).

${ }^{6}$ Note however that, as the inner radii are $\sim 10^{8}-10^{9} \mathrm{~cm}$ for all the pulsars considered, we expect most of the intrinsic light from the disk to be produced in the UV, which is difficult to detect.
} 
Blandford, R.D. \& Romani, R. 1988, MNRAS, 234, 57

Camilo, F., Kaspi, V.M., Lyne, A.G., Manchester, R.N., Bell, J.F., D’Amico, N., McKay, N.P.F., \& Crawford, F. 2001, ApJ, in press astro-ph/0004330

Cannizzo, J.K., Lee, H.M. \& Goodman, J. 1990, ApJ, 351, 38

Caraveo, P.A., Mereghetti, S. \& Bignami, G.F. 1994, ApJ, 423, L125

Chakrabarty, D. \& Kaspi, V.M. 1998, ApJ, 498, 37

Chatterjee, P., Hernquist, L. \& Narayan, R. 2000, ApJ 534, 373

Daumerie, P.R. 1996, PhD Thesis, University of Illinois

Deeter, J.E., Nagase, F. \& Boynton, P.E. 1999, ApJ, 512, 300

Gaensler, B.M. \& Frail, D.A. 2000, Nature, 406, 158

Gold, T. 1968, Nature, 218, 731

Goldreich, P. \& Julian, W.H. 1969, ApJ, 157, 869

Gunn, J.E. \& Ostriker, J.P. 1969, Nature, 221, 454

Gvaramadze, V.V. 2001, astro-ph/0102431

Illarionov, A.F. \& Sunyaev, R.A. 1975, A\&A, 39, 185

Kaspi, V.M., Manchester, R.N., Siegman, B., Johnston, S. \& Lyne, A.G. 1994, ApJ, 422, L83

Lyne, A.G., Pritchard, R.S. \& Graham-Smith, F. 1993, MNRAS, 265, 1003

Lyne, A.G., Pritchard, R.S. \& Smith, F.G. 1988, MNRAS, 233, 667

Lyne, A.G., Pritchard, R.S., Graham-Smith, F. \& Camilo, F. 1996, Nature, 381, 497

Macy, W.W. 1974, ApJ, 190, 153

Manchester, R.N. \& Taylor, J.H. 1977, "Pulsars," Freeman, San Francisco

Marsden, D., Lingenfelter, R.E. \& Rothschild, R.E. 2001a, ApJL, in press, astro-ph/0008300

Marsden, D., Lingenfelter, R.E. \& Rothschild, R.E. 2001b, preprint, astro-ph/0102049

Melatos, A. 1997, MNRAS, 288, 1049

Menou, K., Esin, A.A., Narayan, R., Garcia, M.R., Lasota, J.-P. \& McClintock, J.E. 1999, ApJ, 520,276 
Menou, K., Perna, R. \& Hernquist, L. 2001, ApJ, submitted astro-ph/0102478

Michel, F.C. 1988, Nature, 333, 644

Michel, F.C. \& Dessler, A.J. 1981, ApJ, 251, 654

Michel, F.C. \& Dessler, A.J. 1983, Nature, 303, 48

Mignani, R.P., Mereghetti, S., Gouiffes, C., \& Caraveo, P.A. 1998, The Messenger, 94, 25

Pacini, F. 1967, Nature, 216, 567

Pacini, F. 1968, Nature, 219, 145

Pacini, F. 1971, ApJ, 163, L17

Pacini, F. \& Salvati, M. 1987, ApJ, 321, 447

Perna, R., Hernquist, L., \& Narayan, R. 2000, ApJ, 541, 344

Perna, R. \& Hernquist, L. 2000, ApJ, 544, L57

Shapiro, S.L. \& Teukolsky, S.A. 1983, "Black Holes, White Dwarfs, and Neutron Stars," Wiley, New York

Taylor, J.H., Manchester, R.N. \& Lyne, A.G. 1993, ApJS, 88, 529

Yusifov, I. M., Alpar, M. A., Gok, F., Huseyinov, O. H. 1995, in "The Lives of the Neutron Stars". Proceedings of the NATO Advanced Study Institute. Editor(s), M.A. Alpar, U. Kiziloglu, J. van Paradijs; Publisher, Kluwer Academic, Dordrecht. 